\title{
Dairy intake and type 2 diabetes risk factors: a narrative review
}

\begin{abstract}
Aim

The interest regarding the potential role of dairy products in the prevention of type 2 diabetes (T2D) has emerged. Although results remain mixed, numerous cohort studies have shown that increased dairy consumption is inversely associated with T2D risk. This narrative review evaluates the recent evidence of dairy products intake on T2D risk factors for the prevention of T2D.
\end{abstract}

Material and method

The review is framed within the systematic review and meta-analyses of cohort studies and the individual randomized controlled trials evidence. We searched for existing meta-analyses of cohort studies that addressed the association of dairy intake with incidence of T2D in adults using the MEDLINE (via PubMed) database. For the interventional studies, the literature searched was conducted using MEDLINE (via PubMed) with the following Medical Subjects Heading (MeSH) terms i.e. dairy OR milk OR cheese OR yogurt AND glucose OR diabetes OR insulin resistance OR insulin sensitivity OR pre-diabetes.

Results

Most of the meta-analyses and systematic reviews of the cohort studies point to a reduced risk of T2D with dairy intake of 3 servings per day. This effect was mainly attributed to lowfat dairy, particularly yogurt and cheese. However, there is no evidence in cohort studies that high-fat dairy intake poses any harm.

Conclusion

Dairy products, when incorporated into a healthy diet, likely do not have detrimental effects on glucose-related outcomes. The potential impact of dairy consumption on glucose tolerance tests, insulin levels, insulin sensitivity measures, and plasma glucose levels warrant future investigation.

Keyword: Cardiovascular disease; DGAC-Dietary Guidelines Advisory Committee; Saturated fatty acids; Total energy (TE); USDA 